\title{
Design of an advanced layered composite for energy dissipation using a 3D-lattice of micro compliant mechanism
}

\author{
Vaibhav V. Gokhale, Carl Marko, Tanjimul Alam, Prathamesh Chaudhari, and Andres Tovar \\ Indiana University Purdue University Indianapolis
}

\begin{abstract}
This work introduces a new Advanced Layered Composite (ALC) design that redirects impact load through the action of a lattice of 3D printed micro-compliant mechanisms. The first layer directly comes in contact with the impacting body and its function is to prevent an intrusion of the impacting body and uniformly distribute the impact forces over a large area. This layer can be made from fiber woven composites imbibed in the polymer matrix or from metals. The third layer is to serve a purpose of establishing contact between the protective structure and body to be protected. It can be a cushioning material or a hard metal depending on the application. The second layer is a compliant buffer zone (CBZ) which is sandwiched between two other layers is responsible for the dampening of most of the impact energy. The compliant buffer zone, comprised by the lattice of micro-compliant mechanism, is designed using topology optimization to dynamically respond by distributing localized impact in the normal direction into a distributed load in the radial direction (perpendicular to the normal direction). The compliant buffer zone depicts a large radial deformation in the middle but not on the surface, which only moves in the normal direction. The effect is a significant reduction of the interfacial shear stress with two adjacent layered phases. A low interfacial shear stress translates into a reduced delamination. The ALC's response to the impact is tested by using dynamic finite element analysis. The proposed ALC design is intended to be used for the design of protective devices such as helmets and crashworthy components in vehicle structures.
\end{abstract}

\section{Introduction}

Human injuries are one of the most apprehended issues which demand researchers from medical and engineering fields to do advanced research for ensuring safety of humans. Vehicle crashes are one of the most dreadful sources of injury which demand solutions from scientists continuously. Although many innovative solutions with proved wellness are generated over the years, no product still exists which ensures protection of human beings from severe crashes or impacts. Vehicle crashworthiness is the term which is used to convey the ability of a vehicle structure and its components to protect its occupants. There are four important terms whose values are very important to decide the crashworthiness of the vehicle: 1 . Head Injury Criterion (HIC) 2. Chest Injury (CLIP) 3. Femur Loads 4. Chest deflection [1]. 7/20/2015
On the other hand, head concussions occurring during football games have increased concerns of players. Design of concussion proof or in other words head injury proof helmets is the need of the current era. Studies have revealed that American football helmets do not have capability to limit acceleration and strain on the brain under threshold values [2]. The parameter used for measurement of safety of helmets is also Head Injury Criterion (HIC), which is an integral function of linear acceleration of head in terms of gravity over a certain period of time. Although injury measurements take into consideration various displacements, accelerations and forces to decide the protective ability of the structures, the main design objective of the impact protective structures is to absorb maximum impact energy such that a crash deceleration pulse with an early peak in time and then a gradual decay is obtained.

In most of the energy dampening applications various kinds of foams are implemented to absorb the impact energy. All the research was concentrated on the development of new improved materials for energy absorbing structures. However, advancement in computational capabilities has relaxed the limits for researchers making them able to generate optimized continuum structures for impact energy dissipating applications. Due to the emergence of very efficient and superior additive manufacturing technology processes, manufacturing is also no more a barrier to researchers. Any complex shape can be generated using a variety of materials with the help of additive manufacturing. Topology optimization is the most general form of the structural optimization and has been a favorite choice of researchers for past decade. Different groups have banked on different approaches to perform topology optimization of the continuum structures for impact energy dampening applications.

There are mainly two methods for topology optimization which are Gradient based and non-gradient based topology optimization. Many famous approaches have been used by researchers for topology optimization of energy absorbing structures. Some researchers have used Evolutionary Structural Optimization (ESO) or Bi-directional Evolutionary Structural Optimization (BESO) which is based on the concept of gradually removing unnecessary or inefficient material from a structure to achieve an optimal design $[3,4,5,6]$. Hybrid Cellular Automaton (HCA) is also one of the popular concepts. In HCA only surface elements are allowed to change the density during the structural synthesis process $[7,8,9,10]$. Other multi material topology optimization approaches with the objectives of maximizing strain energy as well as stiffness have implemented micro-inclusion model and implementation of Pareto optimal designs to generate optimized material domains $[11,12]$. 
Traditionally, the aim of the topology optimization was to generate structure with minimum compliance or maximum stiffness with minimum strain energy. However, over the period, topology optimization is used for other applications too. Compliant mechanism synthesis is one of the most potent applications of the topology optimization. In the previous works, some research groups have generated algorithms to generate multi-material domains for energy absorbing structures using this approach $[13,14]$.

The objective of this work is to develop a new Advanced Layered Composite (ALC) design that redirects impact load through the action of a lattice of 3D printed micro-compliant mechanisms providing maximum safety to protective structures such as helmets. The ability of compliant mechanisms to generate the motion at desired locations is effectively used to design our proposed ALC structure. The following sections explain the detailed design procedure in the stepwise manner. The first section introduces conceptual design of compliant mechanism for a complete solid material part of the compliant buffer zone (CBZ) followed by the development of 3D compliant mechanism suitable for additive manufacturing and design of the complete ALC. In the next sections, a benchmark problem is designed which is simple but similar to a real time scenario and the performance of the ALC structure is compared with the traditional existing design. Numerical simulations are performed using dynamic finite element analysis to generate curves of energy, force and displacement of the structure over the time of impact.

\section{Design Development}

In this section we have explained the detailed design procedure for generating the ALC. The focus of the design is on the CBZ as it is the part of the structure responsible for dissipation of most of the impact energy. The CBZ is considered as an array of a large number of cells and each cell can be treated as an independent design domain to generate optimized design. A single cell is further discretized in a number of infinitesimal elements. Each cell is a combination of three material zones: 1. Solid 2.Flexible Foam 3. Void (air). Mathematical models are applied to the elements of cell to generate a topology optimized cellular compliant mechanism (CCM) structure for solid material.

\section{Two-dimensional conceptual design of compliant mechanism using topology optimization}

Compliant mechanism is a structure that takes input force at defined locations and produces displacements at the desired locations in the desired directions. In this work, a monolithic (jointless single structure) compliant mechanism is obtained by using continuum based approach which utilizes topology optimization principles. The formulation of the optimization problem is a multi-objective formulation having the objectives: 1 . Structure should be flexible enough to generate a required motion at the output and 2.The structure should be strong or stiff enough to sustain incoming forces. The displacement at the output port can also be stated as a quantity which is similar to energy called Mutual Potential Energy (MPE) $[15,16]$ or complementary virtual work [17].

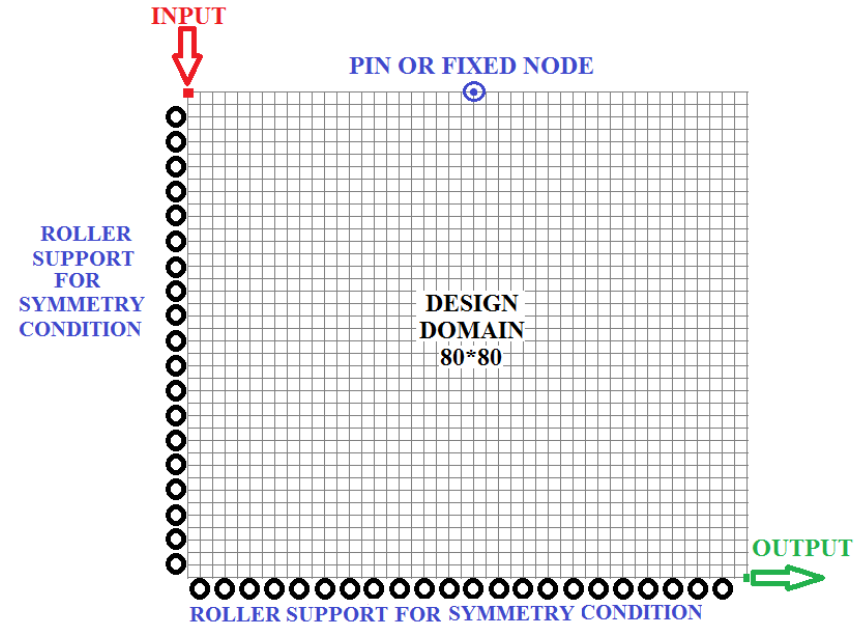

Figure 1.Design domain, boundary conditions and loads for designing a compliant mechanism by topology optimization technique.

This MPE is given as

$$
\operatorname{MPE}(\mathbf{x})=\mathbf{F}_{\text {in }}^{\mathrm{T}} \mathbf{d}_{\text {out }}(\mathbf{x})
$$

where $\mathbf{F}_{\text {in }}$ is the vector of external nodal forces at the input ports, $d_{\text {out }}$ is the vector of nodal displacements at the output ports, and $\mathbf{x}$ is the vector of design variables (e.g. ,material density). MPE is calculated by considering two dummy load cases with one load at the input port and other at the output port. To develop a two dimensional (2D) quarter symmetric conceptual CBZ a $80 \times 80$ domain is considered. The design domain, boundary conditions and loads are defined as shown in the figure 1 . The input load is considered in the vertical direction and the desired output motion is in the horizontal direction. Topology optimization is based on density based approach using regularized solid isotropic material with penalization (SIMP) method and uses optimality criterion optimizer [18]. The optimization problem is:

$$
\begin{array}{cl}
\text { find } & \mathbf{x}=\left[x_{1}, \ldots, x_{e}, \ldots, x_{n}\right]^{\mathrm{T}} \\
\text { maximize } & \operatorname{MPE}(\mathbf{x}) \\
\text { subject to } & v(\mathbf{x})=\mathbf{v}^{\mathrm{T}} \mathbf{x}-\bar{v} \leq 0 \\
& 0 \leq x_{e} \leq 1, \quad e=1, \ldots, n
\end{array}
$$

where $\bar{v}$ is percent of the volume of domain to be occupied by the solid material and it is called as volume fraction. In addition, the algorithm requires regularization parameters such as penalization factor, filter radius and volume fraction. Default values are used as presented in [18].
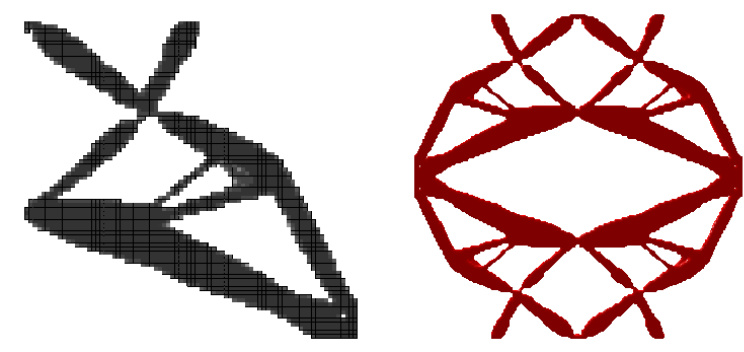

Figure2. Left: Topology optimization of a quarter part. Right: Final 2D structure after post-processing. 
Results of topology optimization may not give the global best value of objective function unless the most appropriate values of these parameters are selected. Hence, several repetitions of the optimization are performed by varying values of manual input parameters. The final structure for the global best value of objective function is as shown on the left of Figure 2. This structure is still not suitable for additive manufacturing and contains problems such as faulty normal, open faces, gaps, etc. Output of topology optimization is post-processed to make it suitable for additive manufacturing by using methods which suggest use of modified filters [19]. The final conceptual design of 2D compliant mechanism after post-processing and taking into account quarter symmetry is shown on the right of figure 2. This is manufactured using the Fused Deposition Modeling (FDM) additive manufacturing technique with some thickness for better visualization and it is as shown in figure 3 .

The conceptual 2D design of compliant mechanism is used to generate the three dimensional (3D) axis-symmetric compliant mechanism using the CAD tools. This new structure has multiple output displacements in a plane which is perpendicular to the axis of symmetry at the mid-height of the structure. The final 3D compliant mechanism is as shown on the left of figure 4 . The areas indicated in blue are fixed to the corresponding adjacent solid layers and areas shown in red can have a sliding contact with the corresponding adjacent layers. This allowance for the relative movement helps in reducing shear stresses in the structure. The remaining space of the CBZ is occupied by a flexible foam and air and hence the entire CBZ looks as shown on the right of figure 4.

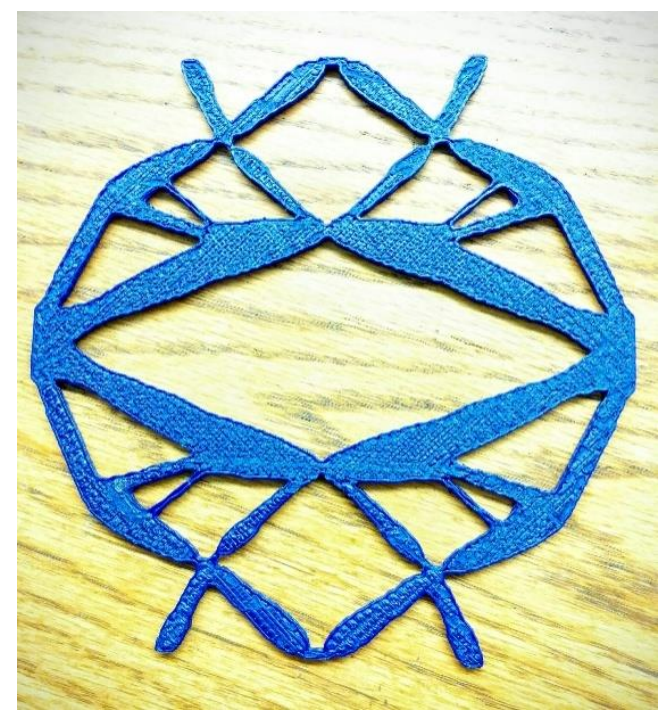

Figure 3.The compliant mechanism generated by additive manufacturing
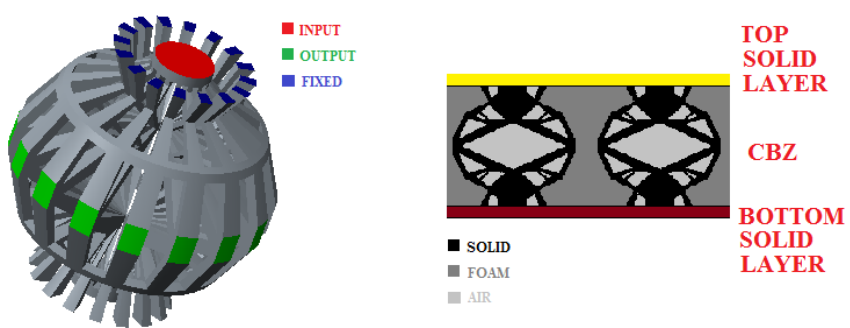

Figure 4.3D compliant mechanism and complete CBZ

\section{Design of a Helmet using ALC}

A complete design of the helmet using the ALC is generated. Figure 14 shows cross-section of the helmet which gives an idea about the arrangement of ALC. Figure 15 shows a part of the helmet which is $3 \mathrm{D}$ printed for the better visualization.

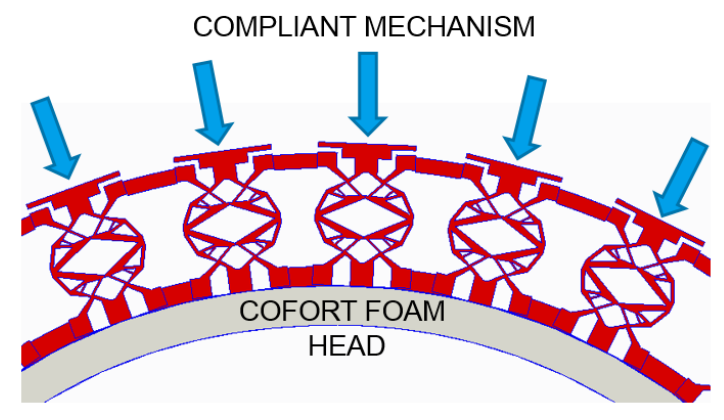

Figure 14.Cross-section of the helmet showing the arrangement of flexible compliant mechanisms domain in ALC.

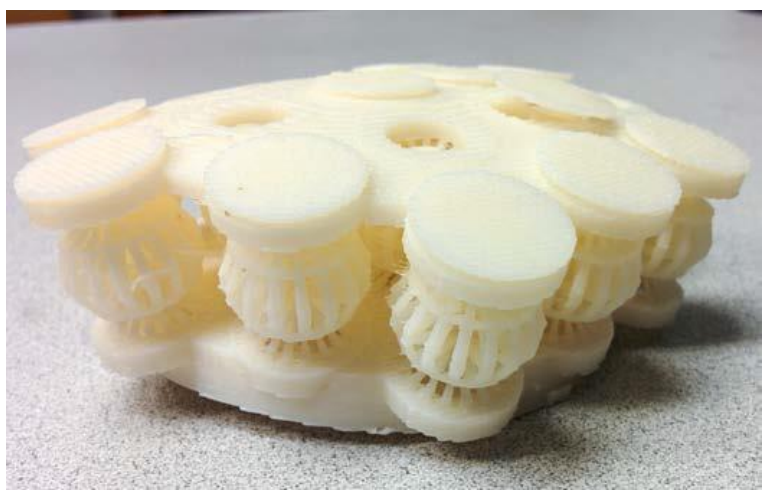

Figure 15.3D printed part of the helmet for better understanding the structure.

The design assembly has the following three parts. 1. Inner comfort foam, 2.ALC, 3.Front shield. Description of the parts is as follows: 


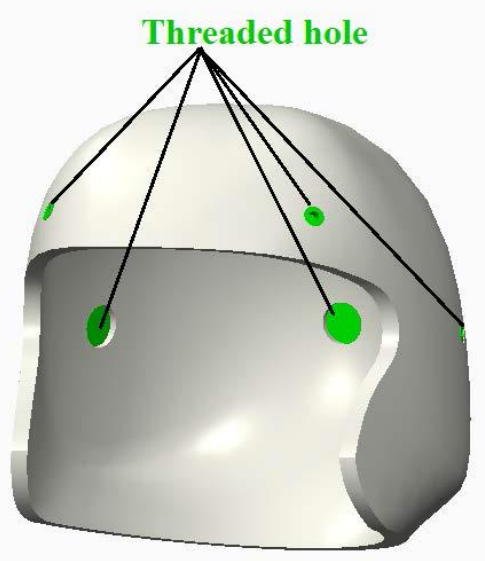

16. The Innermost comfort foam with slots for the threaded hole

Figure

This is the part of the helmet as it exists in the previously described traditional structure which provides comfort to the head. The comfort foam has several slots for accommodating the threaded holes which are used to attach the ALC to the comfort foam. The threaded holes and the screws are made up of Acrylonitrile Butadiene Styrene (ABS) and can be manufactured by a simple FDM additive manufacturing technique. The comfort foam is as shown in the figure 16 .

\section{$A L C$ within the helmet material system}

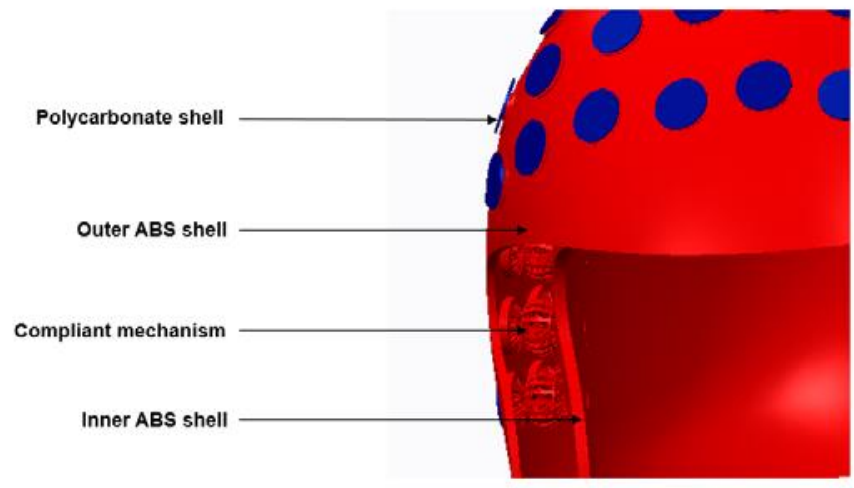

Figure 17.The CAD model of integrated ALC with representation of different regions.

ALC is a continuous structure made of different materials as shown in figure 17. It can be manufactured by advanced techniques such as PolyJet polymer 3D printing. The outer layer of the ALC is further subdivided into two layers, one made of Polycarbonate shell and the other made of ABS shell. The ABS shell is a continuous shell to which fixed regions of all flexible compliant mechanism are attached. The Polycarbonate shell serves as the extended input port of the compliant mechanism. It extrudes out of the ABS shell. When impact occurs impact forces are first impinged on the outermost Polycarbonate shell which further transfers those forces to the designed input port of the flexible compliant mechanism so that compliant mechanism can act as it is designed to act. All the layers of ALC with dimensions and materials are given in the Table 4.

\begin{tabular}{|l|l|l|l|}
\hline No & Description & Material & Layer thickness \\
\hline $1 \& 4$ & Inner and outer layer & ABS & $4 \mathrm{~mm}$ each \\
\hline 2 & Compliant mechanism & $\begin{array}{l}\text { Thermoplastic } \\
\text { Polyurethane } \\
\text { rubber (TPU) }\end{array}$ & $\begin{array}{l}20 \mathrm{~mm} \text { (Average } \\
\text { thickness of the entire } \\
\text { layer) }\end{array}$ \\
\hline 3 & Input port & Polycarbonate & $1 \mathrm{~mm}$ \\
\hline
\end{tabular}

\section{Front protective shield:}

The front protective shield has the conventional design. It is made of aluminum. It can be made by either traditional manufacturing processes or by additive manufacturing. A complete assembly of the helmet is shown in figure 18.
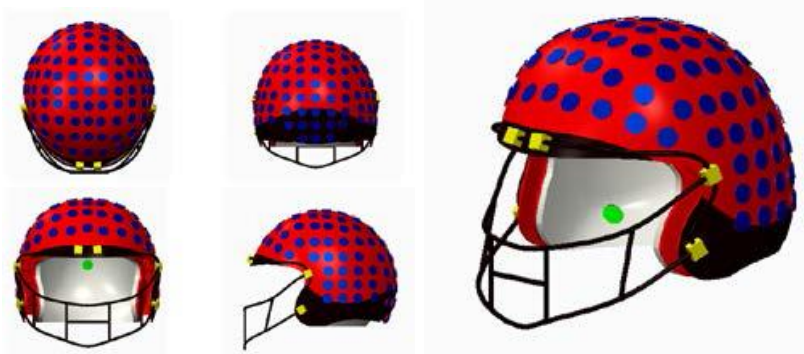

Figure 18.Complete assembly of the helmet. Left: TOP, BACK, FRONT \& SIDE view. Right: 3D view

\section{Performance Evaluation of ALC under Impact}

\section{Finite element analysis model}

In this section, new proposed ALC is compared with the traditional helmet structure for the calculations of performance measures which are directly responsible for the effectiveness of a structure in the application of protection from impact damage. An impact test is performed which is simple, fast and easy to repeat. Non-linear explicit finite element analysis is performed using LS-dyna using non-linear material models. LS-Dyna is an advanced general purpose Multi-physics software package developed by Livemore Software Technology Corporation (LSTC). It is widely used for dynamic simulations all over the world.

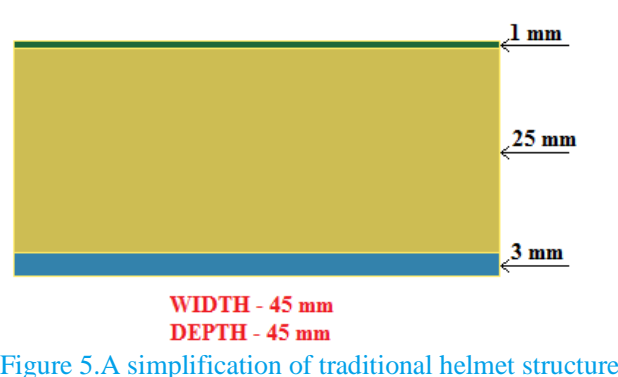

PC outer shell EPS liner foam 
The main function of the helmet is to prevent head from injury during impacts. The different types of helmets like construction helmets, bike helmets, sports helmets, ballistic helmets require different levels of performance standards. The helmet performance is measured in terms of the parameters like Head Injury Criterion (HIC), maximum linear and angular acceleration of the head. The ultimate aim for an effective helmet design is maximizing energy absorption per unit volume though.

The common helmet structure is comprised of three main components: 1. Outer shell, 2.Liner foam, 3.Comfort foam. First two play an important role in protection of the head. For a most common helmet structure, outer shell is made up of polycarbonate (PC) which is a rigid lightweight material. Liner foam is Expanded Polystyrene (EPS) foam which deadens the violence of shock and the vibrations reflected on the head while being compressed [20]. Comfort foam, as its name indicates is only provided for comfort of the head and it is made up of Polyurethane foam (PUR). The average thickness of the PC shell is $1 \mathrm{~mm}$, of the EPS liner foam is $25 \mathrm{~mm}$ and of the PUR comfort foam is $3 \mathrm{~mm}$ [21].

A simple structure with a cubic shape similar to the traditional structure of helmet is generated as shown in figure 5. The CBZ generated in this research is used to replace the EPS liner foam of traditional structure and hence the complete three-layered structure is ALC. Behavior of the two structures are compared under identical impact conditions. The total dimensions of both the structures are $45 \mathrm{~mm} \times 45 \mathrm{~mm} \times 29 \mathrm{~mm}$. The mesh for the compliant mechanism is generated using solid four-noded tetrahedral elements so as to more accurately implement the complex shape. All the other layers in traditional as well as in the ALC structure are composed of solid hexahedral elements. The solid material used for CBZ is NitrileButadiene Rubber (NBR). More details about the material models are given in the table 1. Impacting body is a thin sphere of diameter 100 $\mathrm{mm}$ and is made of Polycarbonate. Velocity of the impacting sphere is $10 \mathrm{~m} / \mathrm{s}$ in the vertical direction in the first case of straight impact and $10 \mathrm{~m} / \mathrm{s}$ at an angle of $45^{\circ}$ with the vertical direction in the second case of oblique impact. The bottom face of the sample is fixed and forces are also measured at the bottom surface of the test sample.

Figure 6 (left) shows the details of test set up for the straight impact case. Whereas, figure 6 (right) shows the details of test set up for the oblique impact case.

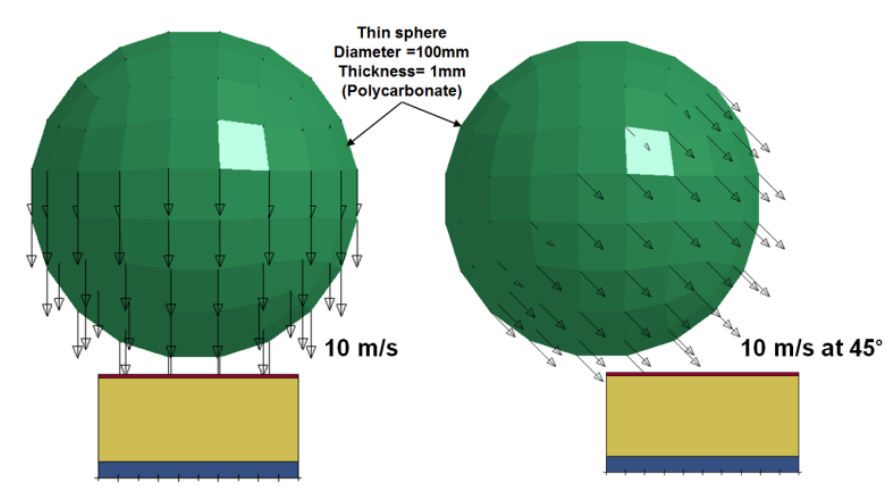

Figure 6. Left: Description of test setup for straight impact. Right: Description of test setup for Oblique impact.

Page 5 of 9
Table 1.Table of material density, Elastic modulus and material models used in LS-Dyna for dynamic finite element analysis

\begin{tabular}{|l|l|l|l|l|}
\hline No & Material & $\begin{array}{l}\text { density } \\
\left(\mathrm{kg} / \mathrm{m}^{3}\right)\end{array}$ & $\begin{array}{l}\text { Elastic } \\
\text { modulus } \\
(\mathrm{Pa})\end{array}$ & Material model \\
\hline 1 & Polycarbonate & 1200 & $2.07 \mathrm{e} 9$ & $\begin{array}{l}\text { 024- } \\
\text { PIECEWISE_LINEAR_PLAST } \\
\text { ICITY }\end{array}$ \\
\hline 2 & EPS foam & 86 & $22.4 \mathrm{e} 6$ & 057-LOW_DENSITY_FOAM \\
\hline 3 & PUR foam & 32 & $0.47 \mathrm{e} 6$ & 057-LOW_DENSITY_FOAM \\
\hline 4 & NBR & 1150 & - & $\begin{array}{l}\text { 027-MOONEY- } \\
\text { RIVLIN_RUBBER }\end{array}$ \\
\hline
\end{tabular}

\section{Straight Impact Test}

\section{Energy absorption}

Figure 7 shows total energy absorption by the helmet structure with ALC and the traditional EPS foam over the time of impact. From the two curves it is seen that the amount of energy absorbed in the form of strain energy is same and almost equal to the kinetic energy of the impacting body. However, energy is absorbed with delayed contact between the impacting body and the protective structure when the ALC is used instead of the traditional helmet structure. This gradual change of energy encourages further to test the ALC using Anthropomorphic Test Devices (ATDs) to see the behavior of protective devices using ALC in future research work. There may be a gradual change in the deceleration pulse of the head with considerable reduction in the peak deceleration and consequently this curve generates a hope that ALC can be considered to be a possible structure which may have potential to enhance the performance of the impact protective devices having an HIC index low enough to overcome brain injuries.

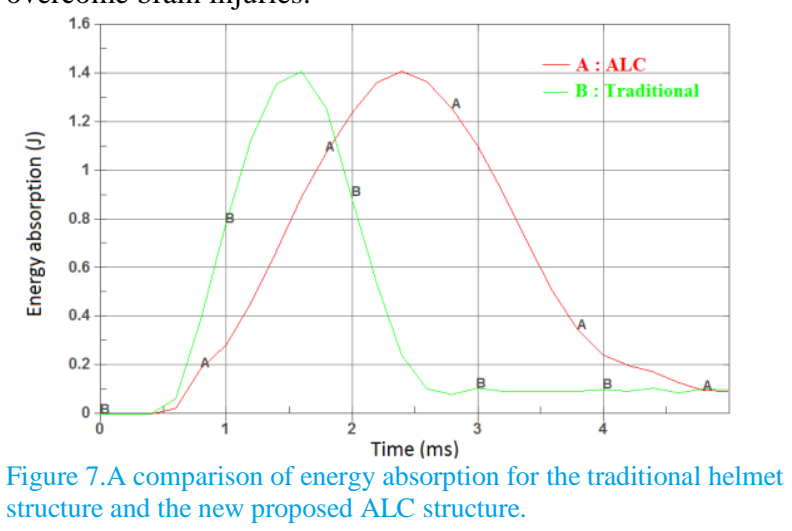

\section{Force in the direction of the impact}

Figure 8 shows the force transmitted by the structures in the direction of impact which is a direct indicator of forces transferred to the head in the real time scenario. The force curve is very smooth for the helmet structure with ALC; but it has sharp variations in case of the traditional structure. Sharp variation of force gives rise to vibrations which are not present when ALC is used. Greater the magnitude of the direct force of the impact transmitted by helmet to the head, greater will be the damage caused to the skull. Hence, we can see from the curve that the ALC may help to minimize the direct damage to the skull due to impact force. Further, it can be fruitful to test the ALC for shock absorption behavior. 


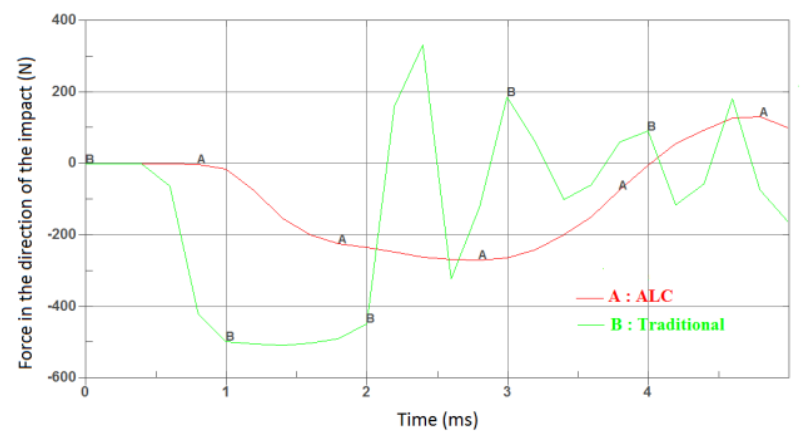

Figure 8.A comparison of transmitted force in the direction of impact for the traditional helmet structure and the new proposed ALC structure.

\section{Displacement or deformation of the structure in the direction of impact}

Figure 9 shows the curve of displacement of the structure in the direction of impact vs. time. The maximum deformation of the structure in the direction of impact is the stopping distance of the impacting body. It is also called as "crush distance" in case of vehicle impacts. Maximum stopping distance is desired for the reduction of accelerations and decelerations caused to human beings and consequentially corresponding injuries. Summary of comparison parameters is given in the Table 2 .

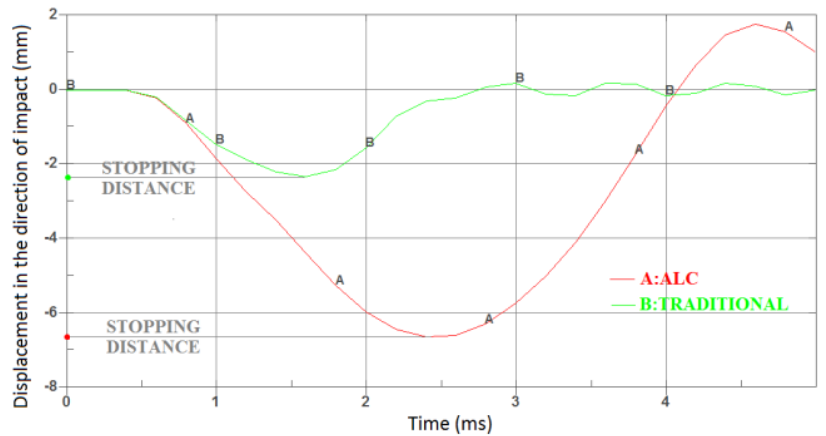

Figure 9.A comparison of transmitted force in the direction of impact for the traditional helmet structure and the new proposed ALC structure.

Table 2. Table of comparison of the traditional structure and proposed ALC structure with respect to various parameters related to the performance measurement.

\begin{tabular}{|l|l|l|l|l|}
\hline No & Parameter & Traditional & ALC & $\%$ change \\
\hline 1 & Mass of the structure (kg) & 0.050 & 0.056 & 12 (increase) \\
\hline 2 & $\begin{array}{l}\text { Peak force in the direction } \\
\text { of impact (N) }\end{array}$ & 500 & 275 & $\mathbf{4 5}$ (decrease) \\
\hline 3 & $\begin{array}{l}\text { Max displacement of the } \\
\text { structure in a lateral } \\
\text { direction perpendicular to } \\
\text { the direction of the impact } \\
\text { (mm) }\end{array}$ & 2.2 & 7.7 & $\mathbf{2 4 0 . 9}$ (increase) \\
\hline 4 & $\begin{array}{l}\text { Sopping distance (mm) } \\
\text { (mm) }\end{array}$ & 2.4 & 6.3 & $\mathbf{1 7 0 . 8}$ (increase) \\
\hline 5 & $\begin{array}{l}\text { Time of contact between } \\
\text { impacting body and } \\
\text { structure (milliseconds) }\end{array}$ & 1.6 & 2.4 & $\mathbf{7 5}$ (increase) \\
\hline
\end{tabular}

Page 6 of 9

\section{Oblique Impact Test}

\section{Energy absorption}

Figure 10 shows curves for energy absorption for both structures. The pattern of curves is similar to that of curves in case of straight impact. However, we can observe that the decrease in the strain energy is even more gradual after the impacting body loses contact with the structure. Hence, the use of this structure might be even more beneficial in case of oblique impact incidents with consideration of the energy absorption pattern.

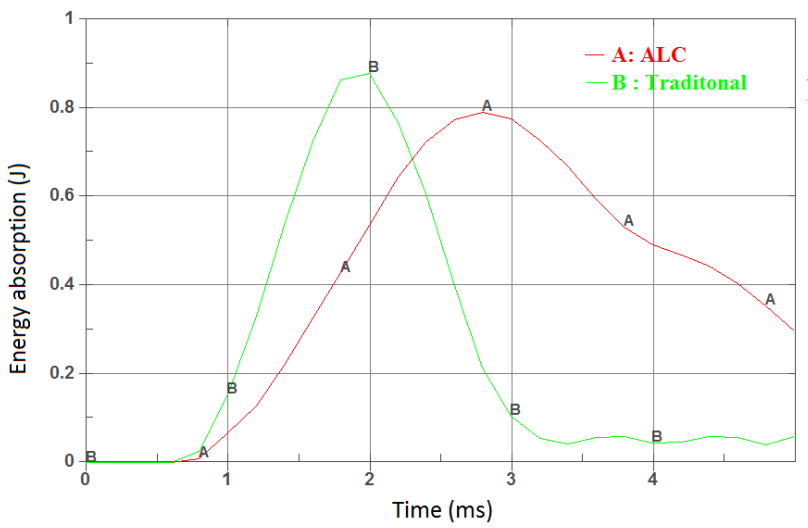

Figure 10.A comparison of energy absorption for the traditional helmet structure and the new proposed ALC structure for oblique impact

\section{Kinetic energy of the impacting body}

Figure 11 shows curves for kinetic energy of the impacting object during impact. We can see that in case of oblique impact, the kinetic energy of the impacting body never decreases to zero that is it never completely stops. The minimum velocity of the impacting object is more when ALC is used. This graph explains why the peak of internal energy is less when proposed ALC is used.

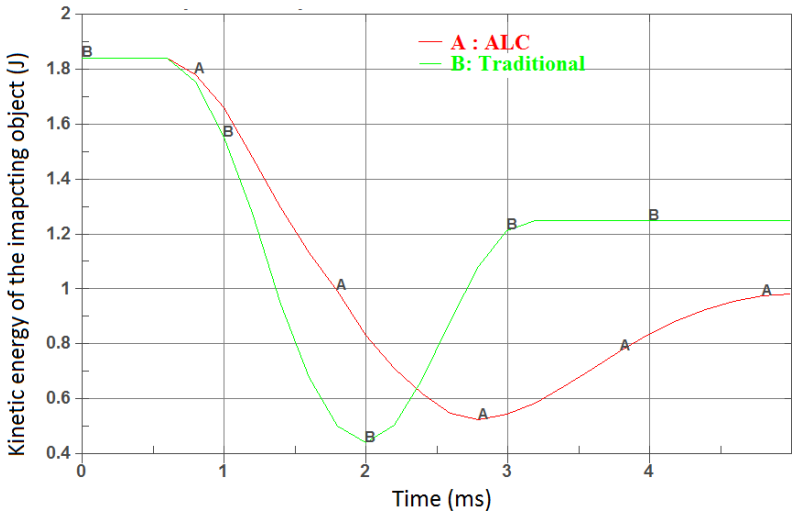

Figure 11.A comparison of dampening of kinetic energy of the impacting body for the traditional helmet structure and the new proposed ALC structure for oblique impact.

\section{Force transmitted in the direction of impact}

Figure 12 shows the curves of force transmitted in the direction of impact. The Force curve is very steep in case of the traditional structure as compared to the ALC. The Peak magnitude of the force is also comparatively low in case of ALC. Hence, future tests on the 
ALC may prove that it has potential to reduce direct damage due to peak impact forces.

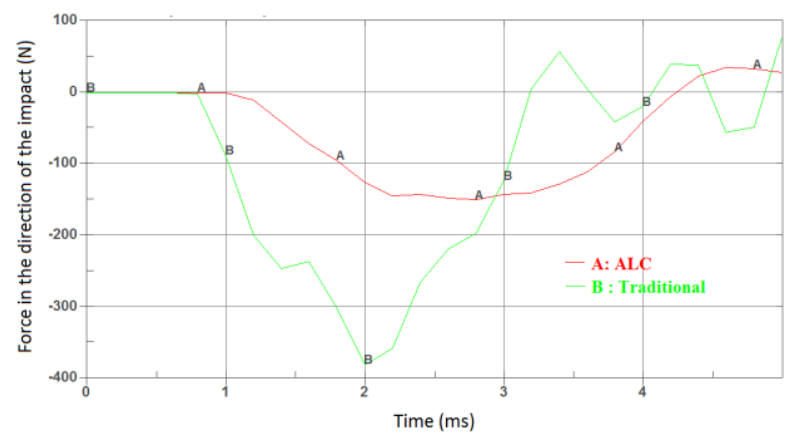

Figure 12.A comparison of force transmitted (virtually to head) by the structure in the vertical direction for the traditional helmet structure and the new proposed ALC structure for oblique impact.

\section{Shear force}

Figure 13 shows the curves of the shear force on the innermost surface of the structure which is a direct indicator of the shear force exerted on head by the helmet. This shear force is the main contributor to the peak angular acceleration of the head. The peak shear force decreases when the structure with ALC is used as compared to the traditional structure. When multiple layers of the ALC are used for protection, the reduced shear forces at the interface can be helpful in preventing delamination between any two layers which consequently may increase the life of the protective structure. Values of the important parameters related to behavior of structures under oblique impacts are compared in the Table 3.

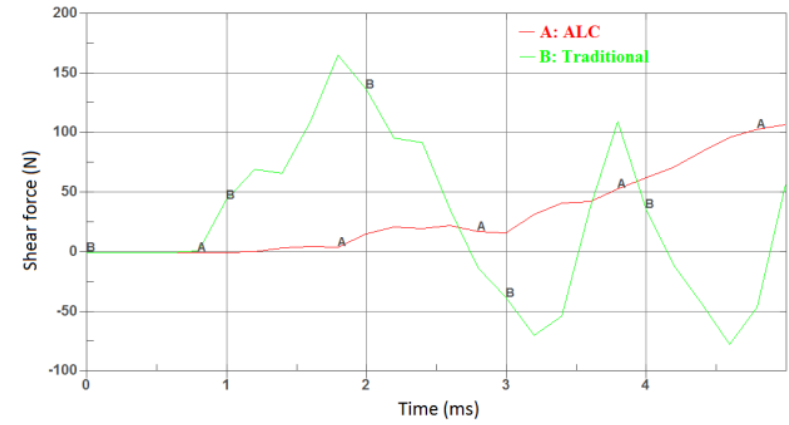

Figure 13.A comparison of shear force transmitted (virtually to the head) by the structure in the horizontal direction (in the direction of horizontal impact velocity component) for the traditional helmet structure and the new proposed ALC structure for oblique impact.

Table 3.Table of comparison of the traditional structure and proposed ALC structure with respect to various parameters related to the performance measurement.

\begin{tabular}{|l|l|l|l|l|}
\hline No & Parameter & Traditional & ALC & $\%$ change \\
\hline 1 & $\begin{array}{l}\text { Peak force in the vertical } \\
\text { direction (N) }\end{array}$ & 380 & 150 & $\mathbf{6 0}$ (decrease) \\
\hline 2 & Max. Shear force (N) & 165 & 107 & $\mathbf{3 5}$ (decrease) \\
\hline 3 & $\begin{array}{l}\text { Time of contact between } \\
\text { impacting the body and } \\
\text { structure (milliseconds) }\end{array}$ & 2 & 2.8 & $\mathbf{4 0}$ (increase) \\
\hline
\end{tabular}

\section{Conclusion}

A new advanced layered composite structural material is proposed by using compliant mechanism synthesis. A simple test is carried by proposing a benchmark example in which structure is subjected to normal and oblique impacts. The results of this analysis show that ALC can give a good damping effect under the impact and interfacial shear forces can be reduced considerably in order to prevent delamination. The damping effect and strength of the composite can be varied by varying component materials of the composite. Each variation of the composite material can be tested using the proposed simple benchmark example test.

The proposed material structure may find application in various areas of energy dampening such as sports-helmets, vehicle crashworthy components, ballistic helmets, etc. The protective devices using ALC can be manufactured using advanced additive manufacturing techniques. In future research, there is a scope to perform crash tests and CAE analysis using Anthropomorphic Test Devices (ATDs) to predict the behavior in impact conditions that are similar to real-time scenarios.

\section{References}

1. Bois, Paul D., Clifford, C.C., Fileta, B.B., Khalil, T.B., King, A.I., Mahmood, H.F., Mertz, J.H., Wismans, J., From "vehicle crashworthiness and occupant protection", American Iron and steel institute, 2004.

2. Honarmandi, P., Sadegh, A.M., Cavallaro, P.V., "Do american football helmets protect players against concussions?", ASME Int Mech Eng Congress Expos Proc, v3B, 2013, doi:10.1115/IMECE2013-64893

3. Huang, X., Xie, Y.M., Lu, G., “Topology optimization of energy absorbing structures", International Journal of crashworthiness, 2007, doi:10.1080/13588260701497862

4. Wang, B.Q., Wang B.L., Huang Z.Y., "Topology optimization for constrained layer damping plates using evolutionary structural optimization method", Advanced Materials Research, v894, p 158-162, doi:0.4028/www.scientific.net/AMR.894.158

5. Stojanov, D., Falzon, B.G., Wu X., Yan W., " An application of bi-directional structural optimization for optimizing energy absorbing structures using a material damage model", Applied mechanics and Materials, v 553, P 836-841,2014, doi:10.4028/www.scientific.net/AMM.553.836

6. Fang, Z., Zheng, L., "Topological optimization for constrained layer damping material in structures using BESO method", Journal of vibration and shock, v33, n8,p 165-170+191, 2014, doi:10.13465/j.cnki.jvs.2014.08.029

7. Patel, N.M., Kang, B., Renaud, J.E., "Crashworthiness design using a hybrid cellular automaton algorithm”, Proc. ASME Des. Eng. Tech. Conf.,v1, p 151-162, 2006, doi:10.1115/DETC200699566

8. Bandi, P., Schmiedeler, J.M., Tovar, A., "Design of crashworthy structures with controlled energy absorption in the hybrid cellular automaton framework", J Mech Des, Trans ASME, 2013, doi:10.1115/1.4024722

9. Guo, L., Penninger, C., Renaud, J.E., Tovar, A., "Strain based topology optimization for crashworthiness using hybrid cellular automata”, Proc. ASME Des. Eng. Tech. Conf., 2009, doi:10.1115/DETC2009-86348

10. Guo, L., Huang, J., Tovar, A., Renaud, J.E., "Multidomain topology optimization for crashworthiness based on hybrid

Page 7 of 9 
cellular automata”, Key Eng Mat, 2011, doi:10.4028/www.scientific.net/KEM.486.250

11. Wildman, R., Gazonas, G., "Multiobjective topology optimization of energy absorbing materials", Struct.

Mutltidiscip. Opt., v51, I1, p 125-143, 2015, doi:10.1007/s00158-014-1117-8

12. Juang, D., Gea, H.C., "Design of energy absorbing structure using topology optimization with multi-material model", Struct. Mutlidiscip. Opt., v32(3), p 251-257,2003, doi:10.1007/s00158006-0011-4

13. Hyland, J.E., Frecker, M.I., Lesieutre, G.A., "Optimization of honeycomb contact-Aided compliant cellular mechanism for strain energy absorption", Proc. ASME Des. Eng. Tech. Conf., v3, 2012, doi:10.1115/DETC2012-71115.

14. Bandi, P., Mozumdar, C.K., Tovar, A., Renaud, J.E., "Design of axially crushing thin-walled square tubes using compliant mechanism approach", Collect Tech Pap AIAA ASME ASCE AHS Struct. Dyn. Mater, 2010, doi: 10.2514/6.2010-2672

15. Frecker, M. I., Ananthasuresh, G. K., Nishiwaki, S., Kikuchi, N., and Kota, S., "Topological Synthesis of Compliant Mechanisms Using Multi-CriteriaOptimization," ASME J. Mech. Des., 119(2), pp. 238-245, 1997, doi: 10.1115/1.2826242

16. Saxena, A., and Ananthasuresh, G. K., "On an Optimality Property of Compliant Topologies", Struct. Multidiscip. Optim., 19(1), p 36-49, 2000, doi: 10.1007/s001580050084.

17. Craig, J.I., Work and Energy Methods for Structural Analysis, Georgia Institute of Technology. p. 26-34.,2005.

18. Liu, K., Tovar, A., "An efficient 3D topology optimization code written in Matlab", Structural and multidisciplinary optimization, pp. 1175-1196, 2014, doi: 10.1007/s00158-0141107-x

19. Zegards, T., Paulino, G. H., "Bridging topology optimization and additive manufacturing", Structural and Multidisciplinary Optimization, 2015, doi: 10.1007/s00158-015-1274-4.

20. Bessai N.,Boudjemaa F., Lounis, M., Baumgartner, D., "Numerical simulation of the mechanical structure of a helmet motorcyclist", Advanced Materials Research, v 856, p 3683722014, doi: 10.4028/www.scientific.net/AMR.856.368

21. Tso-Liang, T., Cho-Liang, L., Van-Hai, N., "Development and validation of finite element model of helmet impact test", Proc. Inst. Mech. Eng. Part L J. Mat. Des. Appl., 2013, doi $: 10.1177 / 1464420712451806$

\section{Definitions/Abbreviations}

CCM

HIC

ESO

BESO

HCA

ALC

2D

Page 8 of 9
3D

CBZ

FDM

CAD

PC

EPS

PUR

ATDs three dimensional

compliant buffer zone

Fused Deposition Modeling

Computer Aided Design

Polycarbonate

Expanded Poly Styrene

Polyurethane

Anthropomorphic Test

Devices

\section{Changes made during revision.}

- $\quad$ All the detected article errors are removed.

- Time unit of all the plots "Time(E-03)(s)" changed to $\rightarrow$ "Time (ms)"

- Introduction

"On the other hand, head concussions occurring in the football have increased concerns of players" changed to $\rightarrow$ "On the other hand, head concussions occurring during football games have increased concerns of players"

"But, advancement in computational capabilities has relaxed the limits for researchers making them able to generate optimized continuum structures for impact energy dissipating applications" changed to $\rightarrow$ "However, advancement in computational capabilities has relaxed the limits for researchers making them able to generate optimized continuum structures for impact energy dissipating applications"

"Different groups have banked on different approaches for generating topology optimization of the continuum structures for impact energy dampening applications" changed to $\rightarrow$ "Different groups have banked on different approaches to perform topology optimization of the 
continuum structures for impact energy dampening applications."

- Benchmark Example - Straight impact- Energy absorption

"But, energy is absorbed with delayed contact between the impacting body and the protective structure when the ALC is used instead of the traditional helmet structure" changed to $\rightarrow$ "However, energy is absorbed with delayed contact between the impacting body and the protective structure when the ALC is used instead of the traditional helmet structure"

- Benchmark Example - Oblique impact- Energy absorption

"But, we can observe that the decrease in the strain energy is even more gradual after the impacting body loses contact with the structure" changed to $\rightarrow$ "However, we can observe that the decrease in the strain energy is even more gradual after the impacting body loses contact with the structure"

- Benchmark Example - Oblique impact- Force transmitted in the direction of impact

"Hence, future tests on ALC may prove that ALC has potential to reduce direct damage due to peak impact forces" changed to $\rightarrow$ "Hence, future tests on the ALC may prove that it has potential to reduce direct damage due to peak impact forces"

\section{- Benchmark Example - Oblique impact- shear force}

Sentence removed $\rightarrow$ "The value of shear force is negligible till impacting body is in contact with the structure"

"This shear force will be responsible for the angular acceleration of the head" Changed to $\rightarrow$ "This shear force is the main contributor to the peak angular acceleration of the head"

"The peak shear force is very less when structure with ALC is used as compared to the traditional structure" Changed to $\rightarrow$ "The peak shear force decreases when the structure with ALC is used as compared to the traditional structure"

"Reduction in shear force at the interface can be helpful in preventing delamination of the layer which may consequently be helpful to increase the life of protective devices" Changed to $\rightarrow$ " When multiple layers of the ALC are used for protection, the reduced shear forces at the interface can be helpful in preventing delamination between any two layers which consequently may increase the life of the protective structure"

- Demonstration of use: Complete design of helmet using ALC - 1.Inner comfort foam

"This is the part of helmet as exists in the previously described traditional structure which gives comfort to head" changed to $\rightarrow$ "This is the part of the helmet as it exists in the previously described traditional structure which provides comfort to the head"

"The comfort foam has several slots for accommodating threaded holes to be used to attach the ALC to comfort foam" changed to $\rightarrow$ "The comfort foam has several slots for accommodating the threaded holes which are used to attach the ALC to the comfort foam"

- Demonstration of use: Complete design of helmet using ALC - 2.ALC

"The outer layer of ALC has been further subdivided into two layers, one made of Polycarbonate shell and other made of ABS shell" changed to $\rightarrow$ "The outer layer of the ALC is further subdivided into two layers, one made of Polycarbonate shell and other made of ABS shell"

- In the last sentence of the abstract we have stated what we mean by protective devices $\rightarrow$ "The proposed ALC design is intended to be used for the design of protective devices such as helmets and crashworthy components in vehicle structures"

Hence, we have used word protective devices in some sentences like "ALC can be considered to be a possible structure which may have potential to enhance the performance of the impact protective devices having an HIC index low enough to overcome brain injuries"

- References section is checked again and edited accordingly. 